\title{
Principal contributing authors
}

\author{
Dr. Alexis McQuitty \\ Jennie Sealy Hospital \\ Department of Anesthesiology \\ 301 University Blvd \\ Galveston, TX 77555 \\ USA
}

Prof. Arnaud Wattiez

IRCAD France-Hôpitaux Universitaires

1 place de l'Hôpital

67091 Strasbourg Cedex

France

\section{Prof. Arnold P. Advincula}

Department of Obstetrics and Gynecology

Columbia University Medical Center

Sloane Hospital for Women at

New York-Presbyterian/Columbia

622 West 168th Street

New York, NY 10032

USA

\section{Prof. Attilio Di Spiezio Sardo}

Department of Public Health

University of Naples "Federico II"

80131 Naples

Italy

\section{Dr. Davor Jurkovic}

University College London Hospitals

Women's Health Division

250 Euston Road

London NW1 2PG

United Kingdom

\section{Assoc. Prof. Dr. Ertan Sarıdoğan}

University College London Hospital

Elizabeth Garrett Anderson Wing

25 Grafton Way

London WC1E 6DB

UK

Prof. Dr. Gokhan Sami Kilic

Urogynecology and Minimally Invasive Gynecology

University of Texas Medical Branch

301 University Boulevard

335 Clinical Science Building

Galveston, TX 77555-0587

USA

\author{
Dr. Ibrahim Alkatout \\ Department of Obstetrics and Gynecology \\ University Hospitals Schleswig-Holstein \\ Campus Kiel \\ Arnold-Heller-Str. 3 \\ Haus 24 \\ 24105 Kiel \\ Germany \\ Prof. Javier Magrina \\ Department of Gynecology \\ Division of Gynecologic Oncology \\ Mayo Clinic Arizona \\ 5779 East Mayo Boulevard \\ Phoenix, AZ 85054 \\ USA

\section{Dr. Jon I. Einarsson} \\ Division of Minimally Invasive Gynecology \\ Brigham and Women's Hospital \\ 75 Francis Street \\ ASB 1-3 \\ Boston, MA 02115 \\ USA
}

Prof. Justin Clark

Birmingham Women's Hospital

Birmingham Women's NHS Foundation Trust and

University of Birmingham

Birmingham, B15 2TG

United Kingdom

Prof. Dr. Kubilay Ertan

Dep. of OB\&GYN

Klinikum Leverkusen

Am Gesundheitspark 11

51375 Leverkusen

Germany

Prof. Dr. Liselotte Mettler

Department of Obstetrics and Gynecology

University Hospitals Schleswig-Holstein

Campus Kiel

Arnold-Heller-Str. 3

24105 Kiel

Germany 
Prof. Marc Possover

International School of Neuropelveology

Witellikerstrasse 40

8032 Zürich

Switzerland

Dr. Mark Hans Emanuel

University Medical Center

Heidelberglaan 100

3584 CX Utrecht

The Netherlands

Dr. Mary Connor

Sheffield Teaching Hospitals

Jessop Wing

Sheffield S10 2SF

UK

Dr. Mohamed Mabrouk

S. Orsola- Malpighi Academic Hospital

Bologna University

Via Massarenti 13

40138 Bologna

Italy

\section{Dr. Mohsen El-Sayed}

Darent Valley Hospital

Darenth Wood Road Dartford

Kent DA2 8DA

UK

\section{Dr. Mostafa A. Borahay}

Department of Gynecology and Obstetrics Johns Hopkins University School of Medicine Johns Hopkins Bayview Medical Center 4940 Eastern Ave

Baltimore, MD 21224-2780

USA

Dr. Natalia Price

John Radcliffe Hospital

Headley Way

Headington

Oxford OX3 9DU

United Kingdom

\section{Prof. Olivier Donnez}

Institut du sein et de Chirurgie gynécologique

d'Avignon (ICA)

Polyclinique Urbain V (ELSAN Group)

Chemin du Pont des Deux Eaux 95

84000 Avignon

France

Prof. Dr. Peter O’Donovan

Bradford Teaching Hospitals

M.E.R.I.T. Centre

Bradford Royal Infirmary

Bradford BD9 6RJ

UK

\section{Prof. Dr. Rainer Kimmig}

Universitätsklinikum Essen (AöR)Klinik für

Frauenheilkunde und Geburtshilfe

Hufelandstraße 55

45147 Essen

Germany

Prof. Rudy Leon De Wilde

Clinic of Gynecology

Obstetrics and Gynecological Oncology

University Hospital for Gynecology

Pius-Hospital Oldenburg

Medical Campus University of Oldenburg

Georgstreet 12

26121 Oldenburg

Germany

Prof. Dr. Sara Y. Brucker

Forschungsinstitut für Frauengesundheit Universitäts-Frauenklinik Tübingen

Calwerstraße 7

72076 Tübingen

Germany

\section{Prof. Sven Becker}

Klinik für Gynäkologie und Geburtshilfe

Klinikum der Johann Wolfgang

Goethe-Universität Frankfurt am Main

Theodor-Stern-Kai 7

60590 Frankfurt

Germany 